\title{
A versatile plug microvalve for microfluidic applications
}

\author{
M. Tahsin Guler ${ }^{\mathrm{a}}$, Pinar Beyazkilic ${ }^{\mathrm{b}}$, Caglar Elbuken ${ }^{\mathrm{b}, *}$ \\ a Department of Physics, Kirikkale University, 71450, Kirikkale, Turkey \\ b UNAM-National Nanotechnology Research Center, Institute of Materials Science and Nanotechnology, Bilkent University, 06800, Ankara, Turkey
}

\section{A R T I C L E I N F O}

\section{Article history:}

Received 5 May 2017

Received in revised form 28 July 2017

Accepted 1 September 2017

Available online 5 September 2017

\section{Keywords:}

Valve

Microvalve

Portable microfluidics

Colorimetric TNT assay

\begin{abstract}
A B S T R A C T
Most of the available microvalves include complicated fabrication steps and multiple materials. We present a microvalve which is inspired from macroplug valves. The plug microvalve is fabricated by boring a hole through a rigid cylindrical rod and inserting it through a microfluidic chip. It simply functions by rotating the rod which aligns or misaligns the valve port with the microchannel. The rod is made up of a rigid material for applying the valve to an elastic polydimethylsiloxane (PDMS) microchannel. The valve can also be used for a rigid channel by inserting the rod into an elastic tubing. Therefore, the presented microvalve can be used for both elastomeric and thermoplastic channels. The plug microvalve can be applied to a prefabricated microchannel and does not require modification of the mold design. We have verified the repeatability and robustness of the valve by repetitive operation cycles using a servo motor. The plug microvalve is adaptable to numerous microfluidic applications. We have shown three modes of operation for the microvalve including fluid flow control across multiple intersecting channels. Integrating the microvalve to some commonly used microfluidic designs, we demonstrated the versatility and the practicality of the microvalve for controlling flow focusing, microdroplet sorting and rapid chemical agent detection. This low-cost microvalve significantly minimizes the prototyping time for microfluidic systems.
\end{abstract}

(C) 2017 Elsevier B.V. All rights reserved.

\section{Introduction}

Fully integrated microfluidic devices require several components such as channels, pumps, mixers and valves. Early years of the birth of microfluidics witnessed the development of these basic components using different methods. In the literature, a variety of microvalves have been reported which employ electro active polymer [1], piezoelectric materials [2], ferrofluids [3], electrostatic actuation [4] or phase change materials such as paraffin wax [5], hydrogel [6], or thermo sensitive materials [7]. There are also single-use burst valves that are normally-closed and opens irreversibly [8]. The passive capillary valves can also be considered as single-use since reactivation of the valve requires complete drying of the chip [9]. One can refer to review articles for numerous other types of microvalves $[10,11]$. Amongst these valves, the pneumatic membrane valve had a profound impact in the field [12]. The high scale integration of these valves allowed exquisite control of fluids in tiny compartments [13]. The multi-layer microfluidic system uses a control layer to adjust the pressure in order to activate or deactivate each valve. The similar doormat valves, which rely on SU-8 molds, operate in a similar way [14]. However, these

\footnotetext{
* Corresponding author.

E-mail address: elbuken@unam.bilkent.edu.tr (C. Elbuken).
}

microvalves require precise multi-layer microfabrication and is not suitable for rapid fabrication. In order to address these challenges the simplistic twist valves have been developed [15]. These valves do not require any off-chip components and can be fabricated relatively easier compared to pneumatic membrane valves. They do not need any pressure or power to retain their state, thus they are applicable for portable applications. However, these twist valves still rely on deflection of a thin PDMS membrane, which is achieved either by twisting a screw or by actuation of a spring plunger [16]. Their fabrication is tedious and include several additional steps such as precise alignment, vertical integration of a screw and curing of a second material to form the threads. It is important to note that both pneumatic membrane valves and twist valves are pinch valves that function based on deflection of an elastic layer which requires microfabrication.

In recent years, microfluidic community has focused more on the application of integrated lab-on-a-chip (LOC) devices with increasing number of researchers from diverse backgrounds. Bringing in new opportunities and new research directions, the increasing demand for more capable systems poses some challenges such as the need for expertise in all the components of an integrated LOC system. It is interesting to note that although micropumps and micromixers are quite standardized and easy-toreach for most researchers, microvalve is still a tricky component especially for people without microfabrication expertise. There is 
still not a gold standard microvalve available in the market. Therefore, there is a gap in the field for low-cost, versatile microvalves that can be fabricated and applied by non-experts.

In this study, we present arguably the simplest type of microvalve that can be exploited in microfluidic systems. We report a plug microvalve that is inspired from macroscale quarter turn ball valves. The plug microvalve is fabricated by boring a hole through a cylindrical rod which is inserted into a hole punched through the microchannel. This valve inherits all the benefits of the twist microvalve; (i) it does not require any change of the original channel design, (ii) it is very low-cost, (iii) it is truly integrated and operates without any off-chip components, (iv) it only requires power during state change. Unlike the twist valve, the plug microvalve can be fabricated in less than 5 min which requires no skillset other than punching and drilling holes. Additionally, the presented valve can be integrated to both elastomeric and thermoplastic microfluidic devices such as polydimethyl siloxane (PDMS), polymethyl methacrylate (PMMA), acrylonitrile butadiene styrene (ABS), polycarbonate (PC) and polystyrene (PS) which is a unique feature. Since it works by completely blocking the microchannel rather than pinching the channel, it works for any channel size or shape (rectangular or round microchannel).

\section{Fabrication and characterization of the plug micro valve}

The operation of the valve is shown in Fig. 1. As seen, the valve is composed of a rod which is inserted to a channel. The rod contains a hole that is aligned with the microchannel in its ON position. Herein, we used polylactic acid (PLA) rods owing to their mechanical properties. PLA is a commonly used biocompatible 3D printing material which is readily available. It has a Young's modulus of 3.5 GPa which provides the rigidity required during the operation of the valve and also ease of fabrication during hole opening.

\subsection{Fabrication}

The plug microvalve is fabricated in four simple steps. First, we cut a piece of rod from PLA filament stock and bore a hole through the radial axis of the rod (Fig. 2a). Then, we bend the tip of the rod $90^{\circ}$ to the direction parallel to the hole using a hot air gun set at $200^{\circ} \mathrm{C}$ (Fig. 2b). This allows ease-of-handling during manual operation. Then, a through hole is opened across the microfluidic chip intersecting the microchannel at the point where we need the valve (Fig. 2c). Finally, the rod is inserted into the hole on a flat surface so that the hole aligns with the microchannel (Fig. 2d).

The through hole of the rod is obtained using a benchtop micro miller (Proxxon MF 70) with tungsten carbide drill bits (Proxxon 28321). PLA rod is fixed into a V-shape groove along a metal fix- ture (Fig. 2a). Drilling is done gradually by $4-5$ rapid up and down motion of the drill bit in order not to increase the temperature of PLA and cause melting. In order to achieve the alignment of the hole with the microchannel in the final device, the distance from the center of the hole to the bottom of the rod should match the height of the microchannel from the bottom of the substrate. In this way, inserting the rod all the way into the microfluidic chip on a flat surface ensures the alignment. The horizontal alignment of the rod with the channel is achieved when punching the hole through the device which is performed under stereo microscope (Fig. 2c). Sample rods with $600 \mu \mathrm{m}$ and $200 \mu \mathrm{m}$ diameter holes are shown in Fig. 2a inset. The dead volumes for these microvalves are $0.48 \mu$ land $0.053 \mu \mathrm{l}$, respectively, which are negligible for most applications.

The rods shown in Fig. 2a are used for soft PDMS microchannels. For microchannels made out of rigid materials such as PMMA, the rod should be elastic to obtain a successful sealing. This is achieved by inserting the PLA rod into a silicone tubing and performing the hole drilling step explained as above. A resultant valve can be seen in Fig. 3c, for which we used a silicone tubing of $0.8 \mathrm{~mm}$ ID and $2.4 \mathrm{~mm}$ OD. (Versilic spx-50). Fig. 3 shows the plug microvalves integrated to a PDMS (a-b) and a PMMA (c-d) microfluidic device. Fig. $3 a$ and $3 c$ show the OFF position of the valve at which the bent section of the rod is perpendicular to the microchannel. Fig. 3b and $3 \mathrm{~d}$ show the $\mathrm{ON}$ position with the red dye solution filling the microchannels. PDMS channels are obtained using conventional soft lithography using SU-8 molds and sealed by bonding to a blank PDMS piece using oxygen plasma activation [17]. PMMA devices were obtained by $\mathrm{CO}_{2}$ laser cutter (Epilog Zing) and sealed by a PMMA piece using double sided tape. After fabrication of the microfluidic devices, the location of the valves were marked using a marker pen. Then, holes are punched through the PDMS or PMMA device using punching or drilling, respectively. The larger the hole through the PLA rod, the more tolerant the system to misalignments.

\subsection{Leakage test}

We tested the sealing of the valve using a pressure pump system (ElveFlow OB1). We connected the inlets of the microchannels shown in Fig. 3 to a DI water supply and gradually increased the inlet pressure up to 2 bar. The valves with $200 \mu \mathrm{m}$ and $600 \mu \mathrm{m}$ hole diameters withstood the maximum pressure of our test setup (2 bar) for both the PDMS device and the PMMA device. During these tests, we have not observed any leakage around the valve thus the plug microvalves were successful in sealing around the hole. It should be noted that successful sealing of the plug microvalve depends on the geometrical parameters and also the chip/rod material elasticity. Although it is challenging to draw a universal

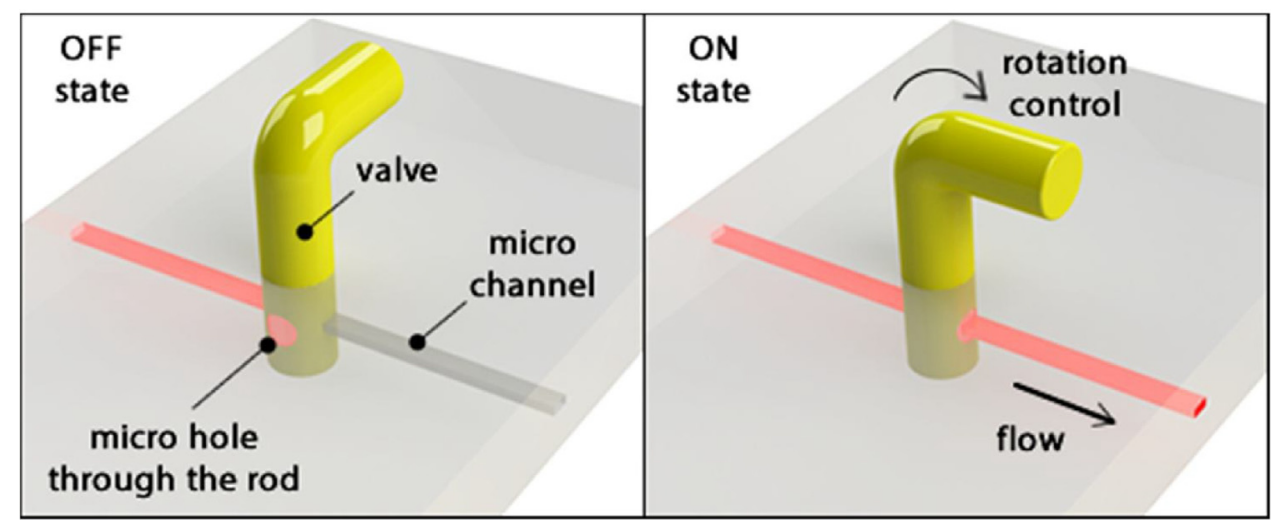

Fig. 1. 3D schematic drawing of the plug microvalve at (a) ON and (b) OFF position. 

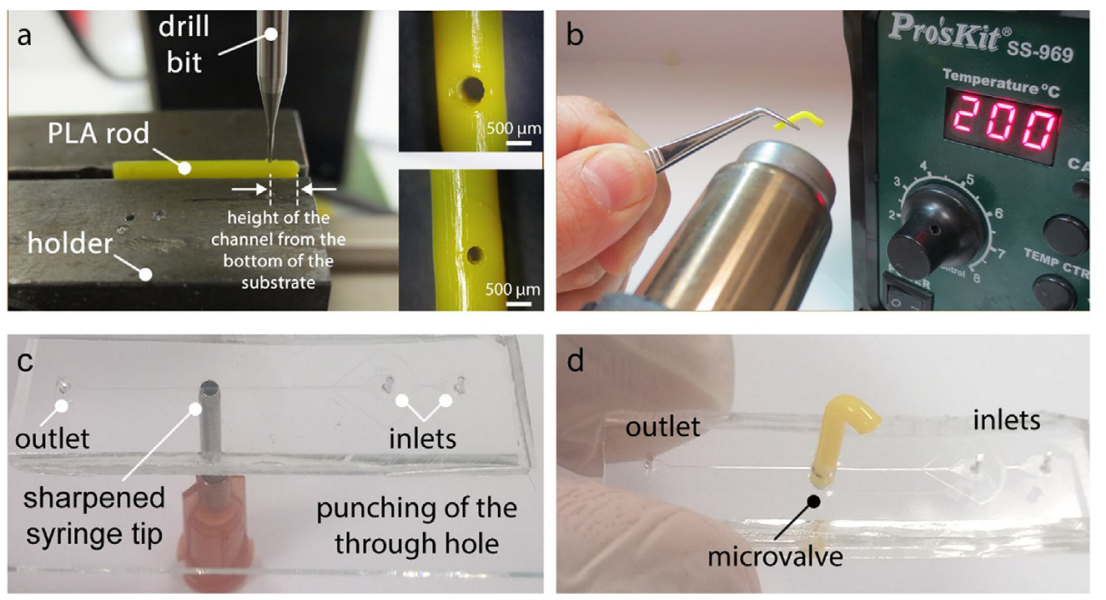

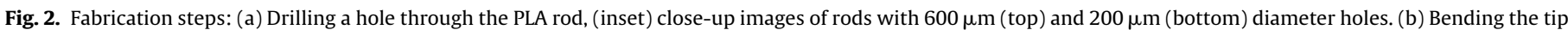

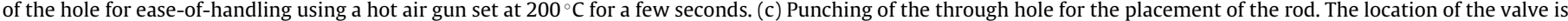
determined at this step and shows the versatility of the plug microvalve. (d) Insertion of the rod to the hole on a flat surface.

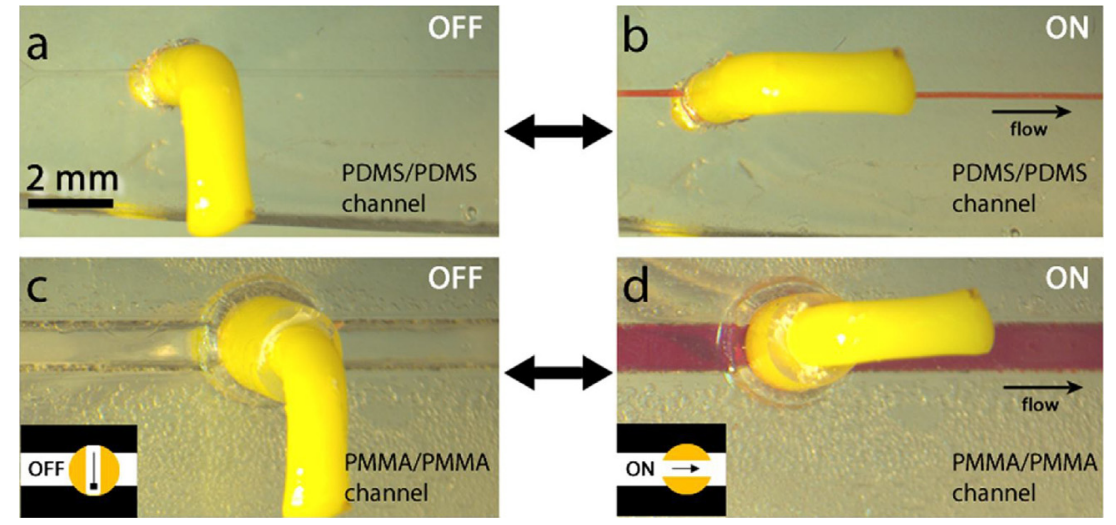

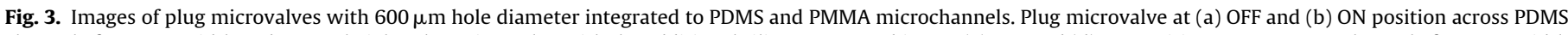

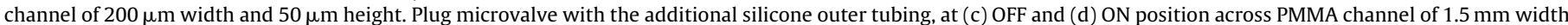
and $200 \mu \mathrm{m}$ height. The fluid is supplied at an inlet pressure of $300 \mathrm{mbar}$ using a pressure pump.

conclusion, from our repetitive experiments with rods and channels of varying sizes, we can provide a design rule of thumb for successful sealing: a) The diameter of the through hole across the rod should be at least twice the size of the channel width. b) The diameter of the rod should be at least twice the size of the hole diameter.

\subsection{Torque test}

The plug microvalve can be operated by hand as shown in the Supplementary movie 1 . We have characterized the torque required for closing/opening the plug microvalve using a digital force meter. A $1.7 \mathrm{~mm}$ diameter rod valve with $600 \mu \mathrm{m}$ hole diameter having $4.4 \mathrm{~cm}$ actuation arm was engaged to the force meter from its farthermost point and pulled retaining the valveintegrated-microfluidic chip. The force value that the valve started to twist was noted. Torque $(\mathrm{g} \mathrm{cm})$ is calculated by multiplying the arm length and the force. The mean values of three successive measurements are shown in Fig. 4.

We measured the torque for PDMS and PMMA microchips of two different thickness. As seen in Fig. 4a, the $4.8 \mathrm{~mm}$ thick PDMS device requires approximately two times the torque required for $2.2 \mathrm{~mm}$ thick PDMS device. It is also evident that torque that is required to activate the valve decreases with increasing hole diameter. $1.7 \mathrm{~mm}$ diameter PLA rod with $600 \mu \mathrm{m}$ diameter hole shown in Fig. 2a was used for PDMS devices punched with 1.2, 1.5 and
$2 \mathrm{~mm}$ diameter punches. We have also tested PDMS microdevices of varying elasticity by varying base polymer/curing agent ratios during fabrication. As seen in Fig. 4a, stiffer PDMS devices (ratio of $5: 1$ ) require less torque for the operation of the valve. It should be noted that the softest PDMS device (ratio of 20:1) with $4.8 \mathrm{~mm}$ thickness was torn off at around $43 \mathrm{gcm}$ during the tests. Therefore, the PLA plug microvalve cannot be used for very elastic microdevices. For such a device, the use of other rod materials can be a solution. For the PMMA device (Fig. 4b), the rod shown in Fig. 3c was used. The results were similar to PDMS device. Increasing hole diameter decreased the required torque while increasing device thickness increased it.

We have also investigated the sealing performance of these microvalves. We have seen that the diameter of the hole punched/drilled through the microfluidic device is a more important parameter than the device thickness and elasticity (studied for only PDMS devices). For the PDMS devices shown in Fig. 4a, the valves integrated with 1.2 and $1.5 \mathrm{~mm}$ diameter punched holes withstood 2 bar inlet pressure. However, when $2 \mathrm{~mm}$ diameter punch was used to make the through hole (which resulted in approximately $1.55 \mathrm{~mm}$ diameter hole waist due to elasticity of PDMS), the valve started to leak at approximately 240 mbar. Similar results were obtained for PMMA devices. For the valves made by $2.51 \mathrm{~mm}$ diameter hole, leakage was observed above $1.2 \mathrm{bar}$, whereas other devices survived the leakage test up to 2 bar. 

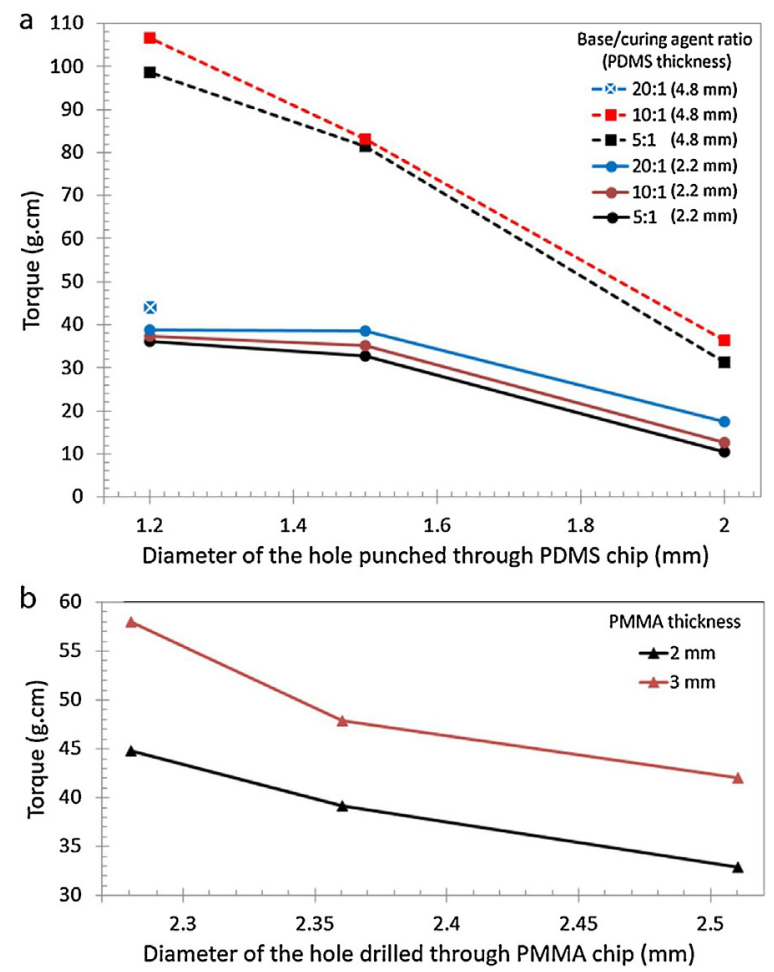

Fig. 4. Experimental torque values measured for $1.7 \mathrm{~mm}$ diameter rod valve actuation for various sizes of holes punched through the device: (a) PDMS device with varying device thickness and base/curing agent ratios, (b) PMMA device with varying device thickness.

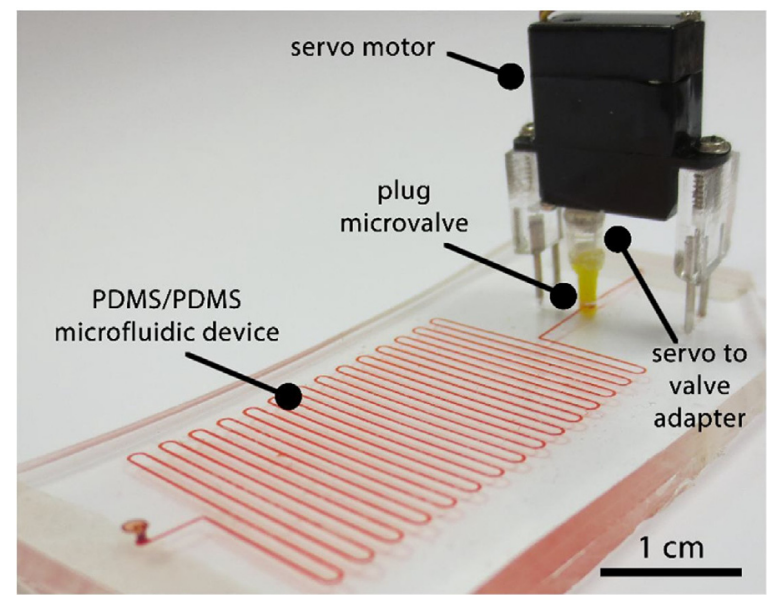

Fig. 5. Automated operation of the plug microvalve using an Arduino controlled servo motor. The fluid is supplied by a pressure pump at an inlet pressure of 300 mbar. The microchannel width and height are 300 and $100 \mu \mathrm{m}$, respectively.

\subsection{Automation and long-term performance}

We have also demonstrated the automated operation of the microplug valve and evaluated its long term performance (supplementary movie 2). For this purpose, we used a servo motor (PZ-15320). In this configuration, we did not bend the tip of the rod and inserted the straight rod with $600 \mu \mathrm{m}$ hole into the servo actuation arm using a custom-made adapter as shown in Fig. 5. The valve was press fit to the servo arm. The motor was fixed on the PDMS device (10:1 mixed, $4.8 \mathrm{~mm}$ thick) for stand-alone operation. It was controlled using an Arduino microcontroller to make a $90^{\circ}$ clockwise rotation and then to go back to home position. The response time of the valve is measured to be approximately $200 \mathrm{~ms}$. We actuated the servo using a square train pulse with a period of $10 \mathrm{~s}$. This

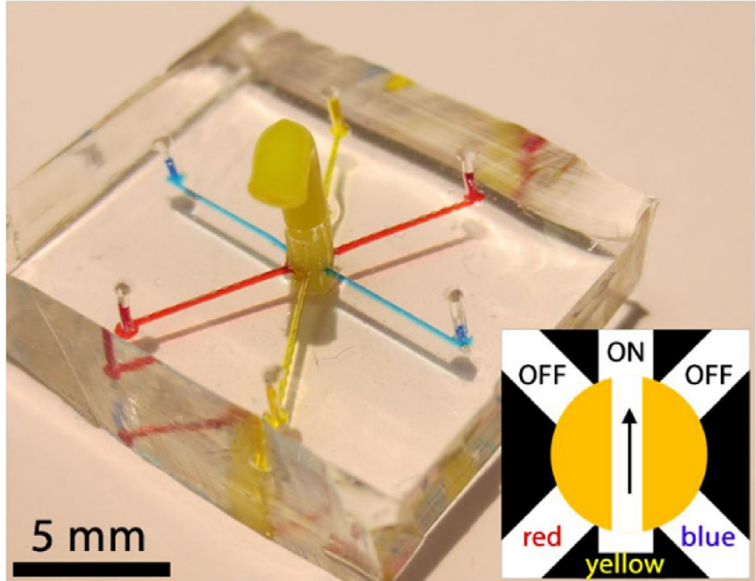

Fig. 6. Control of the flow in multiple microchannels with a single valve. Three straight PDMS/PDMS channels which intersect at a junction where a plug microvalve is placed. In the shown state, valve is ON for the channel with yellow solution and OFF for the remaining two channels. (For interpretation of the references to colour in this figure legend, the reader is referred to the web version of this article.)

resulted in more than 8000 ON/OFF cycles for one day operation. After two days of operation, we have not observed any malfunctioning in the microvalve performance verifying the robustness of the valve. For applications demanding faster switching times, stronger servo motors should be employed.

\section{Modes of operation}

Most microfluidic valves can only be used at a single configuration, which is simply for controlling the fluid flow along a single microchannel. Despite its simplicity plug microvalve can be used in multiple configurations, which is another unique feature of this versatile valve. In this study, we demonstrate three different modes of operation for the plug microvalve using $10: 1$ mixed $4.8 \mathrm{~mm}$ thick PDMS devices.

The basic operation of the microvalve is shown in Fig. 3, which is the ON/OFF fluid control of a single channel. This mode of operation is analogous to single pole single throw (SPST) type electrical switches. As a second mode of operation, the plug microvalve can be used for multiple intersecting channels as shown in Fig. 6. In this configuration the valve turns on one straight channel at a time while closing the others. Fig. 6 shows a PDMS/PDMS microfluidic device demonstrating this feature. Three straight channels of $300 \mu \mathrm{m}$-width and $100 \mu \mathrm{m}$-height were fabricated, which intersect at a single junction. A $1.7 \mathrm{~mm}$ rod diameter valve with $600 \mu \mathrm{m}$ hole is placed at the intersection. Each channel is turned on sequentially and filled with DI solutions of different colors.

In another novel configuration, the plug microvalve is used to control plumbing for single inlet multiple outlet designs. In Fig. 7, a PDMS/PDMS device with a single inlet and two outlets are shown. The microchannel width and height are 300 and $100 \mu \mathrm{m}$, respectively. The channel bifurcation is designed so that the inlet channel first opens to a wide reservoir ( $3 \mathrm{~mm} \times 1 \mathrm{~mm}$ ) which then splits into two channels. The microvalve is placed at this intersection. As seen in Fig. 7, such a configuration provides three different states. First, the two channels are kept OFF, then they are turned ON sequentially allowing fluid flow which is indicated by fluid bulging out at the corresponding outlets. This mode of operation resembles the single pole double throw (SPDT) type electrical switches with an OFF state in between, which is referred as single pole center off (SPCO) configuration. 


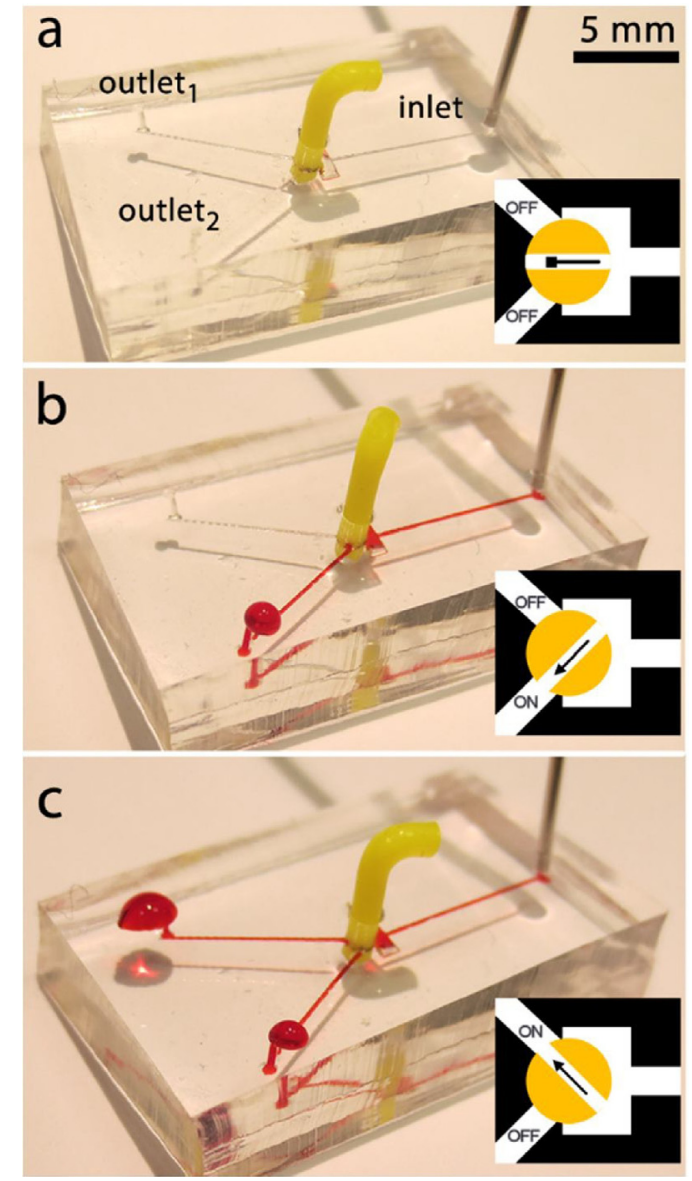

Fig. 7. The use of the plug microvalve for bifurcating junctions. (a) Valve closes both channels. (b) and (c) One of the two outlet channels is turned ON.

\section{Applications and discussion}

To demonstrate the practicality of the valves we have designed three exemplary applications: a flow focusing device, a microdroplet sorting device and a portable TNT detection device. Hydrodynamic focusing, droplet-based systems and portable biosensors/detectors are widely used across several microfluidic applications. The plug microvalves provide key features in all these applications as presented in this section.

First, we fabricated a 2D hydrodynamic flow focusing PDMS/PDMS device with $50 \mu \mathrm{m}$ depth. We used red dye solution to sheath focus yellow dye solution as shown in Fig. 8. The width of sheath flow channel is $100 \mu \mathrm{m}$, the width of the main channel is $200 \mu \mathrm{m}$. We integrated two plug valves with $600 \mu \mathrm{m}$ hole diamater. Fig. 8a shows the valves at their OFF position. For clarity of the channels, first the chip is filled with red dye solution when the valves are ON. Then, the system is tested for the other three states of the two valves. The corresponding microscope images for each state are shown in Fig. $8 \mathrm{~b}-\mathrm{d}$. The valves can be switched ON or OFF without any need for stopping the inlet flows. This allows rapid enabling or disabling of hydrodynamic focusing for quickly switching from focused to unfocused state. We have also tested the partial closure of the valves. Since it is challenging to control the position of the valve for such an operation, it is best to use the presented plug microvalves in the binary mode, i.e. as ON or OFF valves.

For a microdroplet-based fluidic system, we used the valve in a dual channel configuration as shown in Fig. 9. In this way, we were able to sort microdroplets at a T-junction which is previously shown using complicated hydrodynamic control or active sorting methods $[18,19]$. We designed a droplet microfluidic device with a
T-junction generator and a T-junction sorter. Two channels downstream the sorting junction are crossed together. A plug microvalve is placed to this intersection as shown in Fig. 9a,b. In this configuration, the valve opens one channel while closing the other one which causes the microdroplets to be sorted right or left at the sorting junction (Fig. 9c,d). For these experiments we formed water droplets in $100 \mathrm{mPa}$.s silicone oil using inlet pressures of $210 \mathrm{mbar}$ for oil and 200 mbar for water. Such a configuration can be used when microdroplets are required to go through different operations for applications such as biochemical synthesis or single cell studies [20].

Finally, we exploited the microvalves in a portable colorimetric assay for rapid trinitrotoluene (TNT) molecule detection, which is critical for environmental safety monitoring. We used silica nanoparticles which were synthesized via sol-gel method, as the assay reagent. Silica nanoparticles were made fluorescent by loading an aromatic dye called pyrene which shows sensitive fluorescence quenching towards TNT [21]. A PDMS device was designed including a serpentine test channel, a straight control channel (300 $\mu \mathrm{m}$ width, $100 \mu \mathrm{m}$ height) and two reservoirs $(6 \mathrm{~mm} \times 2 \mathrm{~mm}$ ) for qualitative observation (Fig. 10a). Plug microvalves were placed after the reservoirs to control the flow along the control and test channels. Flow is generated by applying negative pressure at the shared outlet using a $0.5 \mathrm{ml}$ syringe. Aqueous solution of silica nanoparticles was introduced to inlet 1 and TNT solution $(0.25 \mathrm{mM})$ was introduced to inlet 2 . Upon the application of negative pressure, the valves were turned ON sequentially. In the serpentine test channel, TNT solution was mixed with nanoparticles using passive diffusion mixing and filled the test reservoir whereas bare nanoparticles followed the straight channel and filled the control reservoir. The test run cycle is shown in Supplementary movie 3. Then, the valves were turned OFF and the device was imaged under a palm-size UV illumination ( $366 \mathrm{~nm}$ ) box for fluorescent detection. The control reservoir gave a bright blue color under UV excitation, whereas the test reservoir was pale blue since fluorescence of silica nanoparticles was quenched by TNT molecules (Fig. 10b) [22].

As a control experiment, the same procedure is repeated with nanoparticle solution replaced with water at inlet 2 . Mixing of nanoparticles with water indicated no influence of dilution on fluorescence signal as shown in Fig. 10c. Quantitative analysis can be performed using the colorimetric signals in the reservoirs. To do this, we converted the colorimetric signals obtained in the UV channel into relative intensity values using Image J. $54.5 \%$ of the fluorescence of silica nanoparticles were found to be quenched by $0.25 \mathrm{mM}$ TNT based on the difference between the intensities of control and test reservoirs (Supplementary Fig. 1). On the other hand, $8.9 \%$ intensity decrease was observed upon mixing with water. This simple device demonstrates the potential of plug microvalve to be used for portable microfluidic detection devices which have numerous environmental and healthcare applications $[23,24]$.

The TNT detection device highlights another critical feature of the plug microvalve. The valves do not require any off-chip components, therefore, after the assay is performed the valves can be turned OFF and the device can be easily transferred to another stage such as into a chamber for tightly controlled incubation or a microscope for imaging. The TNT detection assay shown in this work is a very promising example demonstrating the potential of the plug microvalve to be extended for various microfluidic colorimetric assays where low cost and portable single use devices are needed.

\section{Conclusions}

In this study, we present a plug microvalve for microfluidic devices. The valve can be easily integrated to microfluidic systems 


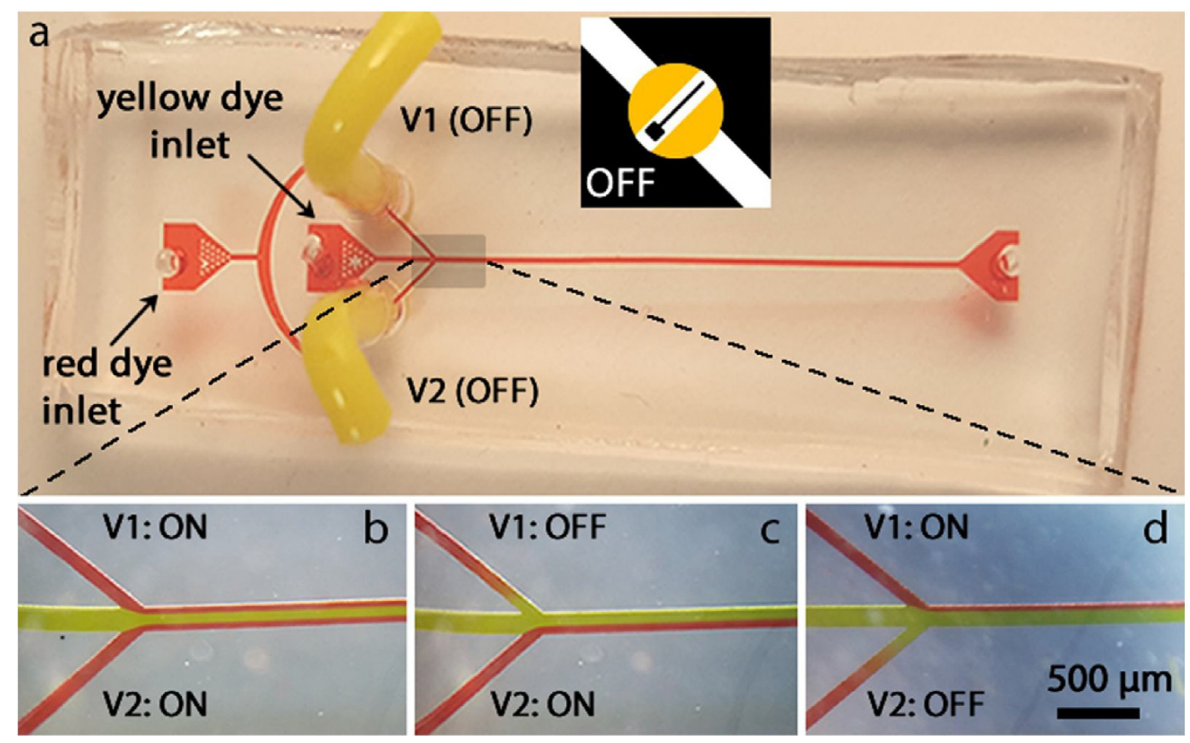

Fig. 8. (a) PDMS flow focusing device with two plug microvalves. (b-d) Three different focusing states achieved by differing positions of the valves. The fluids were supplied at 200 mbar inlet pressure.

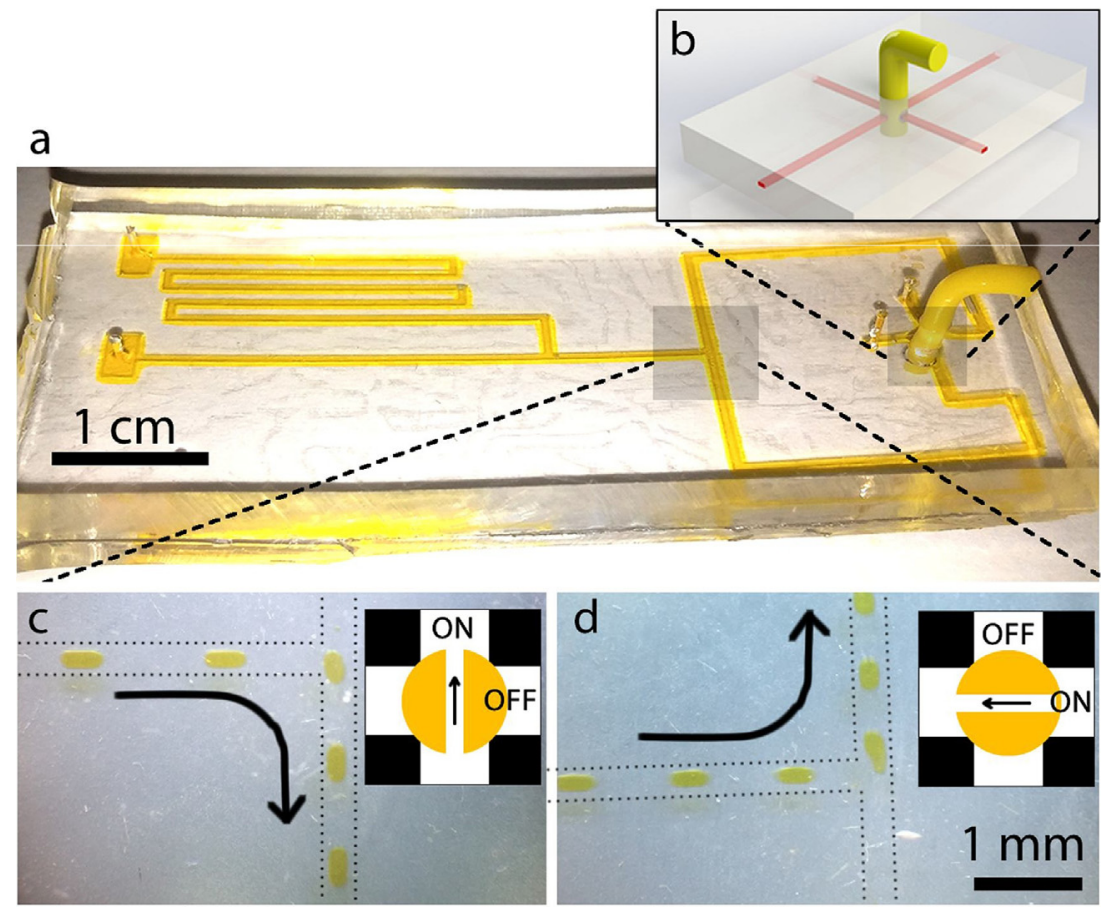

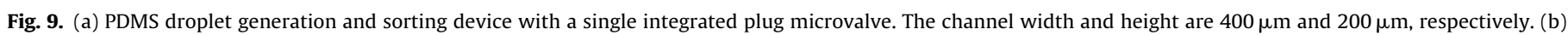
Schematic drawing demonstrating the operation of the valve. Microdroplets are being sorted to the (c) right or (d) left at the T-junction.

without any need for additional mold design. It takes less than $5 \mathrm{~min}$ to fabricate a microvalve and apply it to a channel. We have demonstrated that these valves can be used for both elastic PDMS channels and rigid thermoplastic channels. The plug microvalve is extremely low-cost and suited very well for devices that require a few valves on a microfluidic device. The plug microvalve is also reusable and does not require any off-chip components. The valve proved successful for pressures up to 2 bar and have a long cycle time. The plug microvalve can not only be used for flow control across a single microchannel, but can also be utilized to control fluid flow at multichannel junctions. To demonstrate the versatility of the valves, we used the plug microvalves to control flow focusing, microdroplet sorting and portable chemical agent detection in three exemplary microfluidic systems. The simplicity of these valves make them good candidates for a variety of applications.

\section{Acknowledgments}

This project was partially supported by European Union FP7 Marie Curie Career Integration Grant (no. 322019). P. B. is supported by TUBITAK-BIDEB graduate fellowship. The authors also thank Murat Serhatlioglu for his help on the figures, Ziya Isiksacan and Resul Saritas for critical reading of the manuscript. 


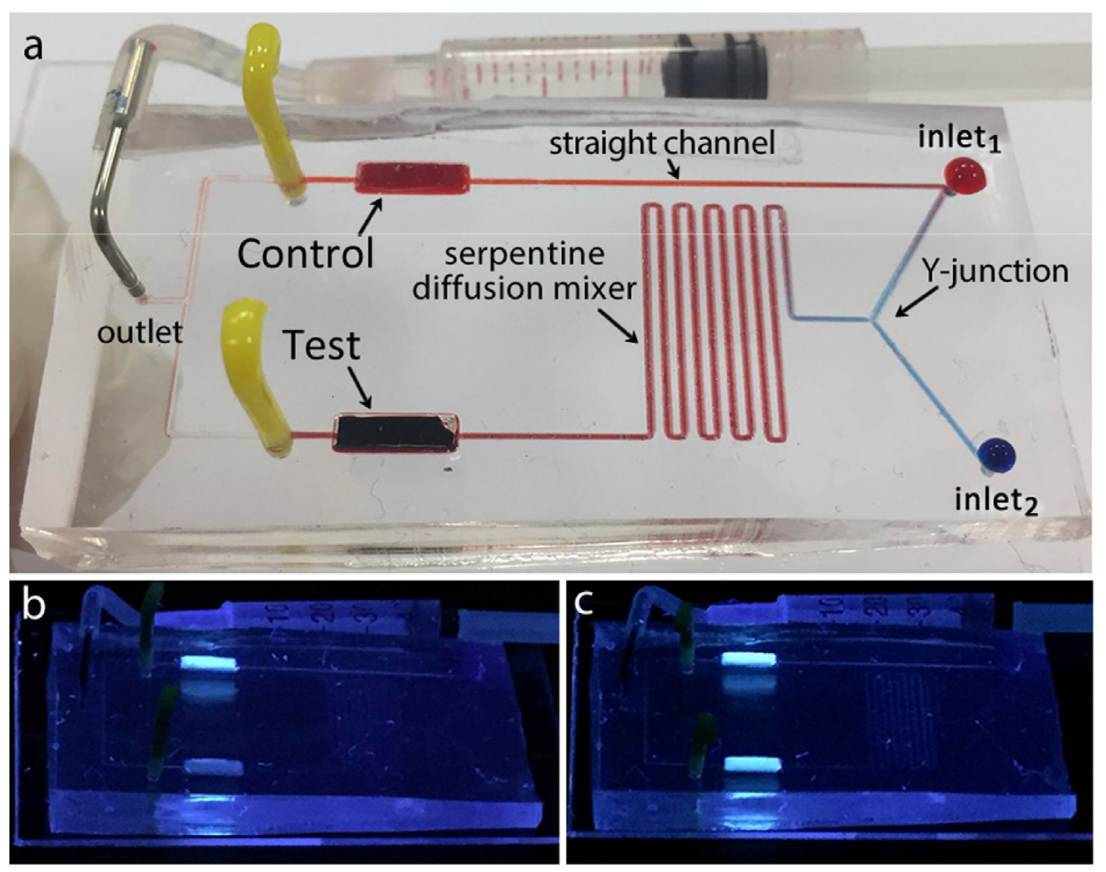

Fig. 10. (a) Plug microvalve-integrated device for rapid and portable qualitative TNT detection. Fluorescent silica nanoparticle and TNT solution were introduced at the two inlets. Mixing of TNT and nanoparticle solutions lead to fluorescence quenching in the test channnel. (b) Device tested with TNT solution under UV light (366 nm) excitation. (c) Control assay run with water instead of TNT solution.

\section{Appendix A. Supplementary data}

Supplementary data associated with this article can be found, in the online version, at http://dx.doi.org/10.1016/j.sna.2017.09.001.

\section{References}

[1] Y. Tanaka, T. Fujikawa, Y. Kazoe, T. Kitamori, An active valve incorporated into a microchip using a high strain electroactive polymer, Sens. Actuators B: Chem. 184 (2013) 163-169.

[2] P. Shao, Z. Rummler, W.K. Schomburg, Polymer micro piezo valve with a small dead volume, J. Micromech. Microeng. 14 (2003) 305.

[3] H. Hartshorne, C.J. Backhouse, W.E. Lee, Ferrofluid-based microchip pump and valve, Sens. Actuators B: Chem. 99 (2004) 592-600.

[4] S.C. Jacobson, S.V. Ermakov, J.M. Ramsey, Minimizing the number of voltage sources and fluid reservoirs for electrokinetic valving in microfluidic devices, Anal. Chem. 71 (1999) 3273-3276.

[5] B. Yang, Q. Lin, A latchable microvalve using phase change of paraffin wax, Sens. Actuators. A: Phys. 134 (2007) 194-200.

[6] M.R. Romero, R.D. Arrua, C.I.A. Igarzabal, E.F. Hilder, Valve based on novel hydrogels: from synthesis to application, Sens. Actuators B: Chem. 188 (2013) $176-184$.

[7] G. Londe, A. Chunder, A. Wesser, L. Zhai, H. Cho, Microfluidic valves based on superhydrophobic nanostructures and switchable thermosensitive surface for lab-on-a-chip (LOC) systems, Sens. Actuators B: Chem. 132 (2008) 431438.

[8] J.T. Santini, M.J. Cima, R. Langer, A controlled-release microchip, Nature 397 (1999) 335-338.

[9] A.-B. Wang, P.-H. Fang, Y.C. Su, Y.-W. Hsieh, C.-W. Lin, Y.-T. Chen, et al., A novel lab-on-a-chip design by sequential capillary-gravitational valves for urinary creatinine detection, Sens. Actuators B: Chem. 222 (2016) 721-727.

[10] A.K. Au, H. Lai, B.R. Utela, A. Folch, Microvalves and micropumps for BioMEMS, Micromachines 2 (2011) 179-220.

[11] K.W. Oh, C.H. Ahn, A review of microvalves, J. Micromech. Microeng. 16 (2006) R13.

[12] M.A. Unger, H.-P. Chou, T. Thorsen, A. Scherer, S.R. Quake, Monolithic microfabricated valves and pumps by multilayer soft lithography, Science 288 (2000) 113-116.

[13] D.W. Lee, Y.-H. Cho, A digital dilution chip using inter-well valves controlled by a ternary microfluidic multiplexer, Sens. Actuators B: Chem. 155 (2011) 380-387.

[14] K. Hosokawa, R. Maeda, A pneumatically-actuated three-way microvalve fabricated with polydimethylsiloxane using the membrane transfer technique, J. Micromech. Microeng. 10 (2000) 415.

[15] D.B. Weibel, M. Kruithof, S. Potenta, S.K. Sia, A. Lee, G.M. Whitesides, Torque-actuated valves for microfluidics, Anal. Chem. 77 (2005) 4726-4733.
[16] Z. Cai, J. Xiang, W. Wang, A pinch-valve for centrifugal microfluidic platforms and its application in sequential valving operation and plasma extraction, Sens. Actuators B: Chem. 221 (2015) 257-264.

[17] D.C. Duffy, J.C. McDonald, O.J. Schueller, G.M. Whitesides, Rapid prototyping of microfluidic systems in poly (dimethylsiloxane), Anal. Chem. 70 (1998) 4974-4984.

[18] T. Glawdel, C. Elbuken, C. Ren, Passive droplet trafficking at microfluidic junctions under geometric and flow asymmetries, Lab Chip 11 (2011) 3774-3784.

[19] H.-D. Xi, H. Zheng, W. Guo, A.M. Gañán-Calvo, Y. Ai, C.-W. Tsao, et al., Active droplet sorting in microfluidics: a review, Lab Chip 17 (2017) 751-771.

[20] H.N. Joensson, H. Andersson Svahn, Droplet microfluidics-a tool for singlecell analysis, Angew. Chem. Int. Ed. 51 (2012) 12176-12192.

[21] P. Beyazkilic, A. Yildirim, M. Bayindir, Nanoconfinement of pyrene in mesostructured silica nanoparticles for trace detection of TNT in the aqueous phase, Nanoscale 6 (2014) 15203-15209.

[22] Y. Wang, A. La, Y. Ding, Y. Liu, Y. Lei, Novel signal-amplifying fluorescent nanofibers for naked-eye-based ultrasensitive detection of buried explosives and explosive vapors, Adv. Funct. Mater. 22 (2012) 3547-3555.

[23] J.C. Jokerst, J.M. Emory, C.S. Henry, Advances in microfluidics for environmental analysis, Analyst 137 (2012) 24-34.

[24] S. Nayak, N.R. Blumenfeld, T. Laksanasopin, S.K. Sia, Point-of-care diagnostics: recent developments in a connected age, Anal. Chem. 89 (2017) 102-123.

\section{Biographies}

Mustafa Tahsin Guler received his B.Sc. degree in Physics from Gazi University and his Ph.D. degree from Kirikkale University Physics. Since 2015, he has been working as a post-doctoral fellow at Bilkent University, National Nanotechnology Research Center - UNAM. His research interests include rapid microfabrication and integrated microfluidic systems.

Pinar Beyazkilic received her B.Sc. degree in Chemical Engineering from Hacettepe University, Turkey in 2011 and MSc degree in the Department of Materials Science and Nanotechnology in 2013. She pursues her Ph.D. degree in Department of Materials Science and Nanotechnology at National Nanotechnology Research Center - UNAM at Bilkent University, Ankara, Turkey. Her research interests include functional surfaces, nanomaterials and biochemical assays.

Caglar Elbuken received his B.Sc. degree in Electrical and Electronics Engineering from Bilkent University, Turkey in 2004 and his Ph.D. degree in Mechanical and Mechatronics Engineering from University of Waterloo, Canada in 2008. He worked as a postdoctoral associate at the Waterloo Microfluidics Laboratory before joining to Abbott Point-of-Care as a senior R\&D scientist. Later, he joined Koc University as a research assistant professor. Since 2012, he is working at Bilkent University, National Nanotechnology Research Center as an assistant professor. His research interests include lab-on-a-chip devices, microdroplet-based microfluidic systems and sensing technologies for portable applications. 